\title{
Rethinking how to get underperforming doctors and medical trainees back on track
}

Cite as: CMAJ 2019 February 11;191:E176. doi: 10.1503/cmaj.109-5711

Posted on cmajnews.com on Jan. 24, 2019.

M any doctors will need remedial help at some point in their careers. But the profession often struggles to confront failure in its ranks, and not much is known about how to get underperforming doctors and medical trainees back on track. Some physicians lose their licences for serious missteps, but more often they undergo remediation, whether that's a quiet word from a colleague or a formal training program mandated by regulators. But according to Dr. Nicola Brennan from the University of Plymouth, "we do not know enough about if and how" these programs work.

Brennan is leading a review, funded by England's National Institute for Health Research, to explore how remediation should work and why in some situations it doesn't. According to Brennan, there may be many reasons a doctor falls short of expectations, including behavioural problems, gaps in knowledge or skills, and underlying health issues.

Remediation programs have come under scrutiny of late, as demands for physician accountability have increased. In Canada, patient complaints against doctors are on the rise. According to the Canadian Medical Protective Association, a small number of doctors is involved in a "larger proportion" of these complaints and require repeated remedial intervention. Similar trends have been noted in the United States, where a 2016 study found that $1 \%$ of physicians paid $32 \%$ of all malpractice claims.

Research on effective remediation is limited and focuses mostly on programs for medical trainees. In a 2018 commentary published in BMC Medical Education, Bennion and colleagues noted that these programs tend to measure success based on trainees passing tests they had failed previously. However, this provides little basis for determining which supports are best for

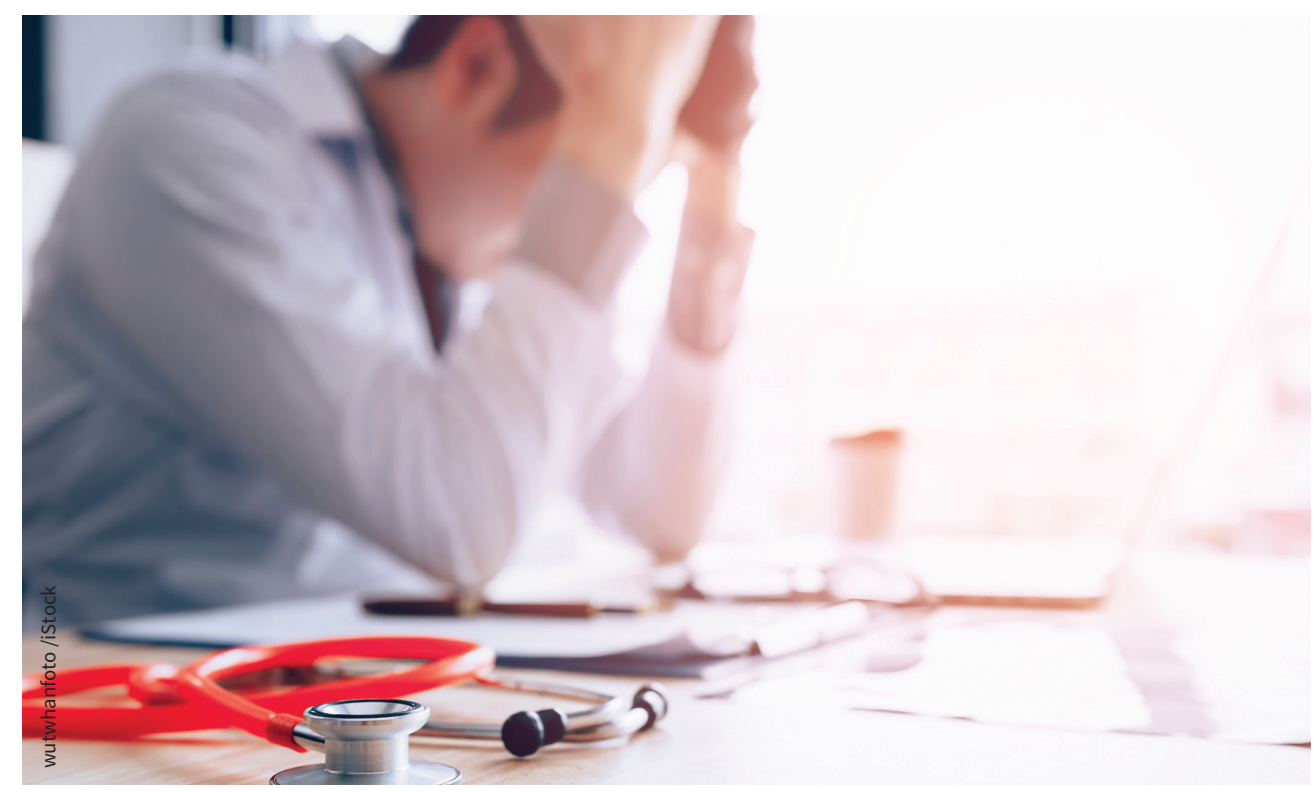

Unclear remediation processes allow underperforming doctors and trainees to slip through the cracks.

which students, or what remediation is most helpful in the long term, they argued.

Medical educators recognize the need to detect and correct trainees who are struggling before their problems compound, but Bennion and colleagues noted that this is rarely done systematically. "Programs and experts sit by the 'sidelines' until there has been a significant failure or a series of failures by an individual student."

Some educators and supervisors are skeptical about the efficacy of remediation, citing lack of standardization and poor outcome data. Among those who expressed reservations in a 2017 University of Auckland study, lack of time and support for remediation were key concerns. In another 2017 paper published in Perspectives on Medical Education, authors noted that many training programs find it difficult to put learners on probation or dismiss them, often for fear of legal reprisal. "The lack of a transparent and articulated remediation policy, combined with insufficient accountability for necessary invocation of the process, can produce a chilling effect," they wrote.

According to Dr. Nadim Lalani, a Calgarybased emergency physician, "the end result is a culture of 'failure to fail' where graduates do not acquire the required skill set." He cited an American survey that found $55 \%$ of internal medicine clerkship directors agreed that grade inflation occurred in their schools, and 38\% noted that students sometimes passed when they should have failed. Lalani attributed these problems to a variety of factors, including a shift toward group work, which can mask individual shortcomings, and a culture in medicine that is often "too busy to teach."

To address these problems, Bennion and colleagues recommended assessing trainees on an ongoing basis across multiple competencies, increasing research on remediation methods, and making more effort to normalize seeking help.

Lauren Vogel, CMAJ 